\title{
Severe Protein-Calorie Malnutrition-Associated Hepatic Steatosis in a Woman Who Had Roux-en-Y Gastric Bypass for Morbid Obesity Thirteen Years Ago
}

\author{
Guriel N. Kim ${ }^{\mathrm{a}}$, Sam Ho ${ }^{\mathrm{b}}$, David Saulino ${ }^{\mathrm{b}}$, Xiuli Liu ${ }^{\mathrm{b}, \mathrm{c}}$
}

\begin{abstract}
Hepatic steatosis is common in everyday liver pathology practice. There are many etiologies leading to hepatic steatosis. These etiologies include metabolic syndrome, alcohol, medications, monogenetic disease, infectious diseases, and malnutrition. Correct diagnosis of underlying etiology through clinicopathological correlation is key to adequate treatment and optimal outcome for the patient. In this case report, we describe severe protein-calorie malnutrition as an etiology for hepatic steatosis in a middle-aged woman who presented with lethargy, low body mass index $\left(15.8 \mathrm{~kg} / \mathrm{m}^{2}\right)$, abdominal distention and bilateral lower extremity edema, hyperammonemia, and hypoalbuminemia, 13 years after Roux-en-Y gastric bypass for morbid obesity. Laboratory tests revealed hyperammonemia, hypoalbuminemia, and low ceruloplasmin levels. Hemodynamic measurement demonstrated high hepatic venous pressure gradient of $12 \mathrm{~mm} \mathrm{Hg}$. Transjugular liver biopsy showed moderate macrovesicular steatosis, mild siderosis, and abundant lipofuscin but no evidence of fibrosis, cirrhosis, or steatohepatitis. This patient was treated with lactulose and enteral feeding, however, the patient died of progressive liver failure 3 weeks after admission. We also review relevant literature to help diagnose protein-calorie malnutrition (kwashiorkor) and hepatic steatosis as a possible late complication of Roux-en-Y gastric bypass. In patients with hepatic steatosis, encephalopathy, hyperammonemia and portal hypertension, malnutrition should be considered as an etiology and diagnosed with a synthesis of clinical, pathological, and laboratory information. Kwashiorkor is a severe disease and should be treated promptly as it may be fatal as in our case.
\end{abstract}

Keywords: Liver; Steatosis; Protein-calorie malnutrition; Hyperammonemia; Hypoalbuminemia; Roux-en-Y gastric bypass; Morbid

Manuscript submitted March 10, 2021, accepted March 24, 2021

Published online April 21, 2021

${ }^{a}$ College of Public Health, University of Florida, Gainesville, FL 32610, USA bepartment of Pathology, Immunology, and Laboratory Medicine, College of Medicine, University of Florida, Gainesville, FL 32610, USA

${ }^{c}$ Corresponding Author: Xiuli Liu, Department of Pathology, Immunology, and Laboratory Medicine, College of Medicine, University of Florida, 1600 SW Archer Rd, PO Box 100275, Gainesville, FL 32610, USA.

Email: xiuliliu@ufl.edu

doi: https://doi.org/10.14740/gr1378 obesity; Ceruloplasmin; Kwashiorkor

\section{Introduction}

Hepatic steatosis is common in everyday liver pathology practice with most cases due to metabolic syndrome. Other etiologies include starvation, cachexia, protein malnutrition, total parenteral nutrition, drugs/medications, alcohol, and infections (including coronavirus disease 2019 (COVID-19)) [1]. Rare monogenic diseases should also be considered [2]. In pregnant women, the differential diagnosis for hepatic steatosis also includes acute fatty liver of pregnancy and HELLP (hemolysis, elevated liver enzymes, low platelet count) syndrome. Bariatric surgery has been used for several decades to treat morbid obesity, and more recently, type 2 diabetes mellitus/metabolic syndrome [3-6]. Jejunocolic and jejunoileal bypass surgeries for morbid obesity have been abandoned due to high mortality and high rates of hepatic complications such as acute liver failure [3]. In current practice, the most commonly performed bariatric surgeries are gastric banding, sleeve gastrectomy, duodenal switch, and Roux-en-Y gastric bypass. Among them, a common one is Roux-en-Y gastric bypass. Hepatic complications after Roux-en-Y gastric bypass are rare. When they do occur it is usually within the rapid weight loss period shortly after surgery (ranging from 7 months to 33 months postoperatively) $[4,5]$, presenting as wasting, lethargy, hypoalbuminemia, and hyperammonemia. Histology in liver failure associated with obsolete (i.e., jejunocolic and jejunoileal bypass) bariatric surgery includes steatosis and, in some cases, steatohepatitis and variable fibrosis as reported in older literature $[3,4,7]$. However, pathology in liver failure associated with modern bariatric surgery (i.e., Roux-en-Y gastric bypass) has not been well illustrated in the literature.

Herein, we report one case of severe protein-calorie malnutrition (kwashiorkor) and hepatic steatosis in a woman who had Roux-en-Y gastric bypass 13 years ago for morbid obesity. The patient presented with acute liver failure, hyperammonemia, acute hepatic encephalopathy, and acute urinary tract infection. We detail the clinical presentation, biochemical abnormalities, hemodynamic measurements, and liver histology. In addition, we also discuss relevant differential diagnoses for hepatic macrovesicular steatosis. Further, we also perform lit- 
erature review on this rare complication after Roux-en-Y gastric bypass.

\section{Case Report}

The patient was a middle-aged woman who had chronic depression and chronic back pain. She had been opioid dependent but had transitioned to using kratom 4 years ago. She stopped using kratom 2 years ago and began using alcohol. She quit drinking alcohol 2 months ago after being told that she had elevated liver tests. Over the last 2 months, she experienced progressive deterioration with increasing confusion. She also started having momentary confusion and memory difficulties. Her husband also noticed black stools and poor oral intake over the week prior to admission. She was then brought to a local hospital where she was found to be anemic and to have bilateral pleural effusions, pericardial effusion, ascites, and imaging consistent with hepatic steatosis. She was transferred to our hospital for further treatment.

Her past medical history was remarkable for Roux-en-Y gastric bypass 13 years ago for morbid obesity (body mass index (BMI) $38 \mathrm{~kg} / \mathrm{m}^{2}$ ) with complications (hypertension). Per intraoperative note, her Roux limb was $110 \mathrm{~cm}$. She had a normal metabolic profile prior to bariatric surgery with only slightly elevated total cholesterol of $211 \mathrm{mg} / \mathrm{dL}$. Her blood pressure was normalized after gastric bypass and her hypertension medications were discontinued. She had no family history of liver diseases; both parents, three siblings, and four children were healthy. She had no protein-rich food aversion. Her only medication at admission was quetiapine $50 \mathrm{mg}$ per day.

Physical exam noted an overall sickly appearance with jaundice, scleral icterus, and lethargy. Also noted on the exam was tachycardia with pulse of 115 beats per minute, abdominal distention, and bilateral lower extremity edema. Her body temperature was $36.7^{\circ} \mathrm{C}$, respiratory rate was 18 breaths per minute. Multiple scabbed round wounds were present throughout her skin primarily located in the buttocks, sacral coccygeal area, to hips. The patient's BMI was $15.8 \mathrm{~kg} / \mathrm{m}^{2}$ at admission. Laboratory testing revealed the patient was anemic (hemoglobin $7.8 \mathrm{~g} / \mathrm{dL}$ ) and thrombocytopenic (platelet count $96 \times$ $\left.10^{3} / \mu \mathrm{L}\right)$. Total protein $(3.7 \mathrm{~g} / \mathrm{dL})$ and albumin $(<1.5 \mathrm{~g} / \mathrm{dL})$ were markedly decreased with elevated ammonia $(136 \mu \mathrm{mol} / \mathrm{L})$ and mildly increased international normalized ratio (INR) (1.4). Her blood urea nitrogen (BUN) was low at $5 \mathrm{mg} / \mathrm{dL}$ (normal $6-21 \mathrm{mg} / \mathrm{dL}$ ). In addition, her alanine transaminase (ALT), aspartate transaminase (AST), and alkaline phosphatase (ALP) levels were normal. Hepatitis A, B, C workup was negative. Autoantibodies (antinuclear antibody (ANA), anti-smooth muscle actin (ASMA), anti-liver/kidney microsomal-1 (LKM$1)$, and anti-mitochondrial antibody (AMA)) were negative. Her ceruloplasmin was $<6 \mathrm{mg} / \mathrm{dL}$ (normal $18-58 \mathrm{mg} / \mathrm{dL}$ ) and plasma copper was low at $28.4 \mu \mathrm{g} / \mathrm{dL}$ (normal $80-155 \mu \mathrm{g} / \mathrm{dL}$ ). Her transferrin was less than $75 \mathrm{mg} / \mathrm{dL}$ (normal 200 - $360 \mathrm{mg}$ / dL). Her vitamin B6, 25-hydroxy vitamin D, and zinc levels were low. Urine culture was positive for Escherichia coli and Klebsiella pneumoniae. Urine protein was negative. Her urine drug screening at admission was unremarkable. Her laboratory test results are summarized in Table 1. Outside head computed tomography (CT) was negative for intracranial hemorrhage. Outside chest and abdominal CT revealed bilateral pleural fluid and ascites. Abdominal ultrasound revealed a nodular liver with ascites. Her hepatic venous pressure gradient (HVPG) was $12 \mathrm{~mm} \mathrm{Hg}$. Esophagogastroduodenoscopy (EGD) revealed features consistent with Roux-en-Y gastric bypass with normal anastomosis and normal mucosa in the examined efferent limb of jejunum. Colonoscopy was unremarkable. Biopsies of the terminal ileum and colon did not reveal abnormalities. Transjugular liver biopsy was performed to identify the etiology of abnormal liver function and cirrhosis.

The liver biopsy was adequate for interpretation. The hepatic lobules demonstrated moderate, primarily macrovesicular steatosis (65\%) most prominently in zones 1 and 2 (Fig. 1). In addition, many ceroid material-containing Kupffer cells were present (Fig. 2). Abundant brown pigment was noted in the periportal hepatocytes (Fig. 2) and centrilobular hepatocytes. Prussian blue iron stain revealed the coarse brown pigment noted in the periportal hepatocytes to be iron, with an estimated mild degree of concentration (Fig. 3). The brown pigment present in the centrilobular area did not stain with Prussian blue and was most likely lipofuscin (Fig. 4). No classic ballooned hepatocytes or definite Mallory-Denk bodies were identified. No overt glycogenosis was noted. There was no significant portal inflammation, lobular inflammation, hepatocyte necrosis, or obvious cholestasis. Portal-based bile ducts were normal and did not show evidence of significant injury. Portal veins and hepatic arteries were likewise unremarkable. Central veins were morphologically unremarkable. There was no evidence of nodular regenerative hyperplasia. No significant fibrosis was noted on routine hematoxylin and eosin (H\&E) stain or on trichrome stain. No diagnostic cytoplasmic alpha-1 antitrypsin inclusions were identified on the periodic acid Schiff (PAS) or PAS with diastase digestion (PAS-D) stains.

Based on morphological features, supported by clinical and laboratory findings, this patient was diagnosed as having moderate macrovesicular steatosis secondary to severe protein-calorie malnutrition (kwashiorkor), likely secondary to Roux-en-Y gastric bypass. The patient was treated with antibiotics (ceftriaxone) for acute urinary tract infection, lactulose for hyperammonemia, and enteral tube-feeding for malnutrition. However, the patient died of progressive liver failure 3 weeks after admission.

\section{Discussion}

The liver plays an essential role in metabolism including fat homeostasis. Many etiologies and insults leading to excessive intake, increased synthesis, decreased consumption, and/ or decreased secretion can lead to an accumulation of fat in hepatocytes (i.e., hepatic steatosis). The differential diagnosis for hepatic steatosis is broad. Excessive calorie intake and decreased calorie utilization as a result of lack of physical activity lead to obesity and nonalcoholic fatty liver disease with or 
Table 1. Laboratory Results

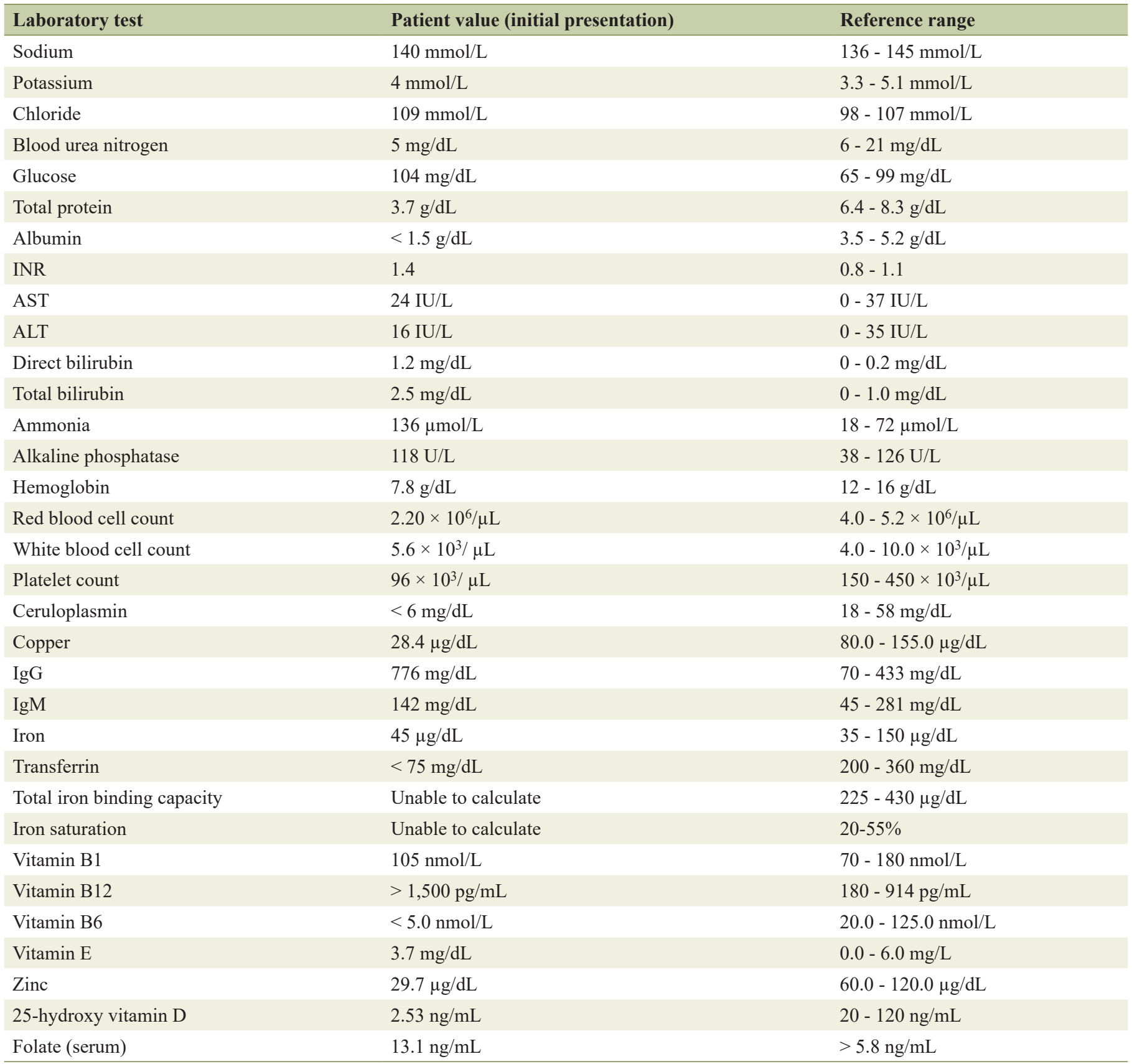

INR: international normalized ratio; ALT: alanine transaminase; AST: aspartate transaminase; Ig: immunoglobulin.

without diabetes. Excessive alcohol use can also lead to steatosis. Medications and other drugs can also lead to steatosis. In addition, patients with many rare genetic diseases (such as Wilson disease, abetalipoproteinemia, familial hypobetalipoproteinemia, glycogen storage diseases, and lipodystrophy) can develop steatosis. Although acute fatty liver of pregnancy and HELLP syndrome more often present with foamy degeneration/microvesicular steatosis, a small percentage of cases can have macrovesicular steatosis.

Many of the above-mentioned etiologies are easily ruled out based on clinical information. For example, excessive calorie intake/obesity/diabetes (nonalcoholic fatty liver disease) or alcohol use are ruled out in our case because the patient had a low BMI and abstinence from alcohol for at least 2 months. Medication-induced liver injury is also unlikely, as the only medication she was noted to be on prior to admission was quetiapine, a drug not particularly associated with hepatic steatosis. Acute fatty liver of pregnancy and HELLP can be ruled out because the patient was not pregnant.

Because our patient had Roux-en-Y gastric bypass for 


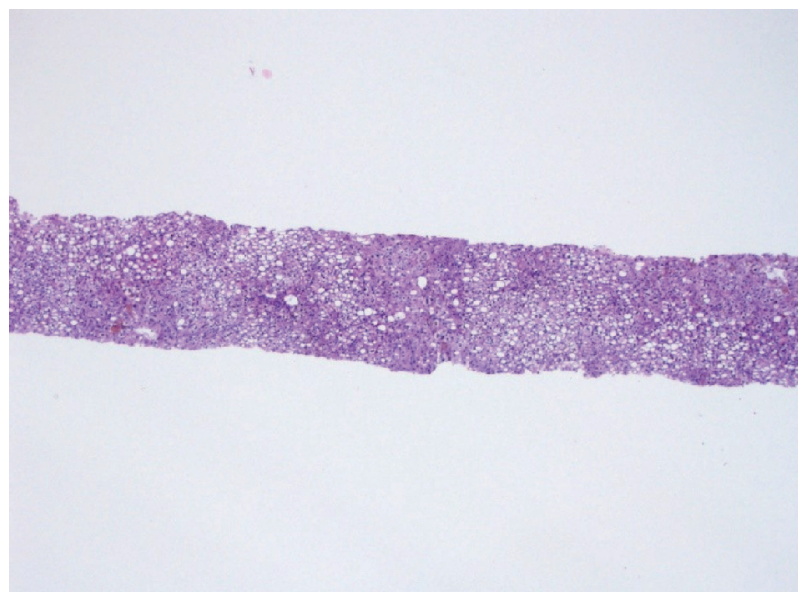

Figure 1. Histology of liver biopsy. Macrovesicular steatosis occupying about $65 \%$ biopsy volume (H\&E stain, $\times 100) . H \& E$ : hematoxylin and eosin.

morbid obesity, a possibility of bariatric surgery-associated liver failure and hepatic steatosis should be considered. Historically, bariatric surgeries such as jejunocolic bypass and jejunoileal bypass were known to cause liver failure [3]. The liver failure in this setting generally occurs within a few months after surgery during the rapid weight loss period and more often in patients with more profound obesity than this patient. Further, these patients usually present with lethargy, jaundice, and hypoalbuminemia. They typically have mildly elevated liver enzymes. Hepatic complications after jejunocolic bypass and jejunoileal bypass have high mortality. Liver (acquired by biopsy or at autopsy) consistently reveals steatosis (often severe) and cholestasis, with or without necrosis. For this reason, and others, jejunocolic bypass and jejunoileal bypass were abandoned in the 1950s and 2000s, respectively. Currently performed types of bariatric surgeries are gastric banding, sleeve gastrectomy, Roux-en-Y gastric bypass and duodenal switch. In the USA, Roux-en-Y gastric bypass is one of the most com-

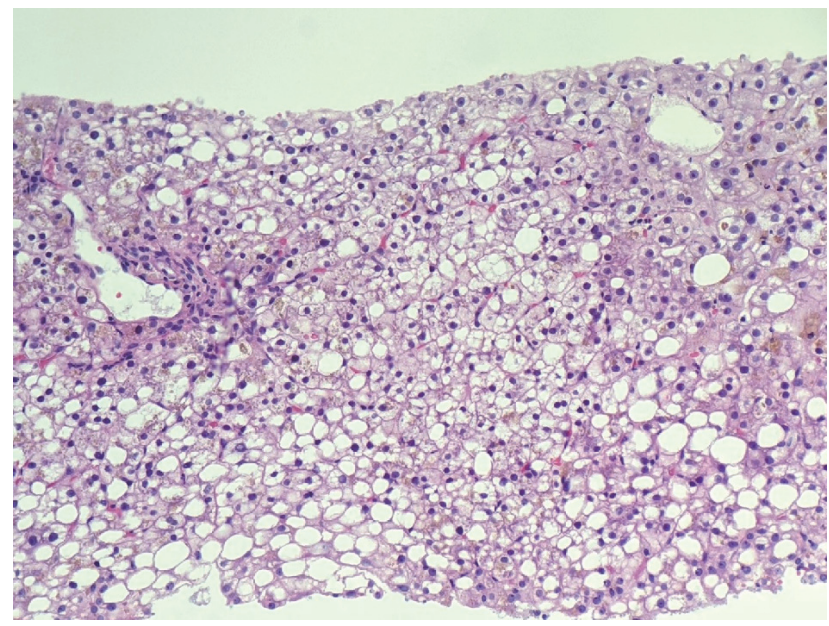

Figure 2. Macrovesicular steatosis, abundant intra-hepatocellular brown pigment, and ceroid material-containing Kupffer cells (H\&E stain, $\times 200)$. H\&E: hematoxylin and eosin.

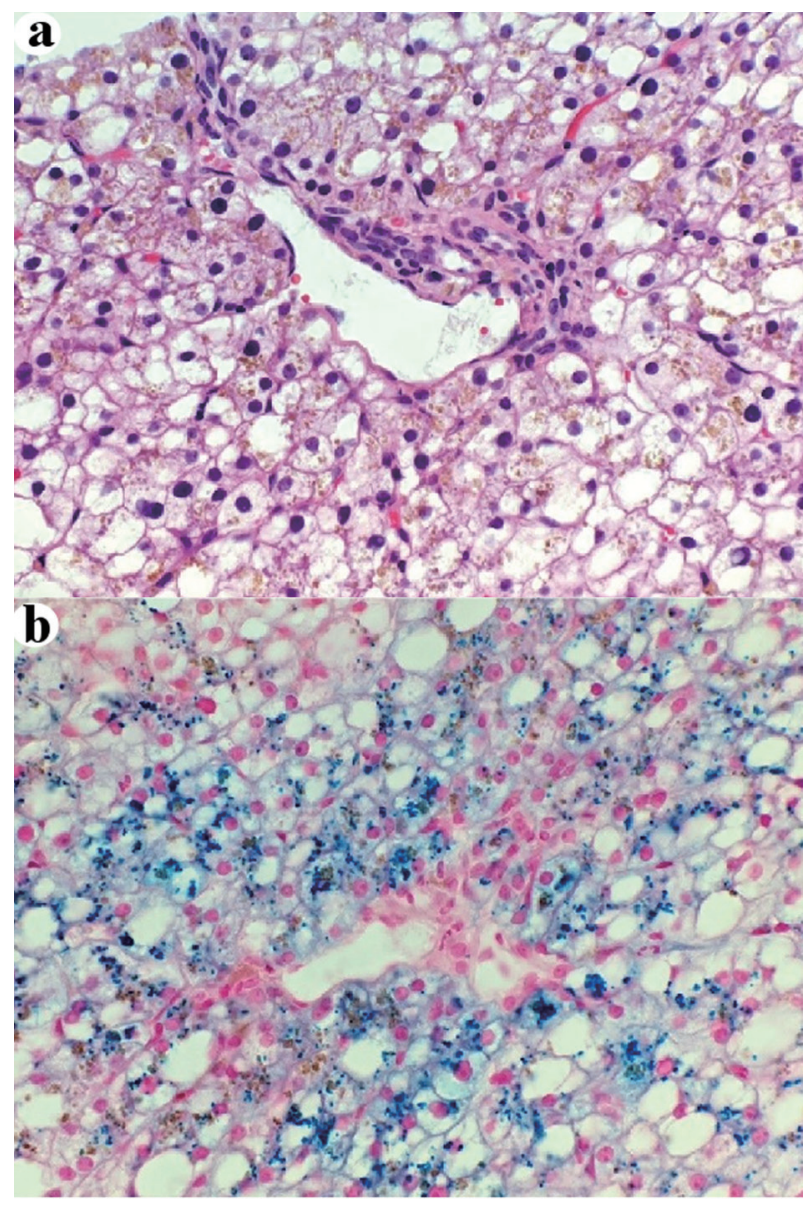

Figure 3. Some periportal intra-hepatocellular brown pigment is iron (a) H\&E stain, $\times 400$. (b) Prussian blue stain, $\times 400$. H\&E: hematoxylin and eosin.

mon bariatric surgeries. Hepatic complications after Roux-en$\mathrm{Y}$ gastric bypass are rare. A few case reports and small case series of liver failure after gastric bypass exist in the literature (Table 2) $[4,5,7]$. Patients were often middle-aged woman (34 - 54 years old) and had gastric bypass for morbid obesity (pre-surgery BMI $42-86 \mathrm{~kg} / \mathrm{m}^{2}$ ). The interval between the surgery and liver failure was between 7 and 33 months. Patients typically had significant weight loss (25-62\% of total weight) and presented with poor oral intake, wasting, and lethargy. All patients had profound hypoalbuminemia $(1.0-2.7 \mathrm{~g} / \mathrm{dL})$, hyperammonemia $(86-224 \mu \mathrm{mol} / \mathrm{L})$, and elevated INR (1.7 to $>$ 7). Elevated HVPG (25-30 mm Hg) was noted in all patients tested. It was suggested by some authors that an extended Roux limb (more than $150 \mathrm{~cm}$ in length) was a risk factor for hepatic complications [5]. Three out of five patients died and liver biopsy revealed severe steatosis with mild fibrosis in one case and steatosis, steatohepatitis, and cirrhosis in two cases. Our patient had a similar clinical presentation: wasting, low BMI $\left(15.8 \mathrm{~kg} / \mathrm{m}^{2}\right)$, lethargy, hypoalbuminemia, hyperammonemia, elevated INR, hepatic steatosis, and elevated HVPG. It is possible that the hepatic steatosis and liver function abnormalities in this case may be secondary to bariatric surgery by the same mechanisms as these previous cases, although the timeframe 

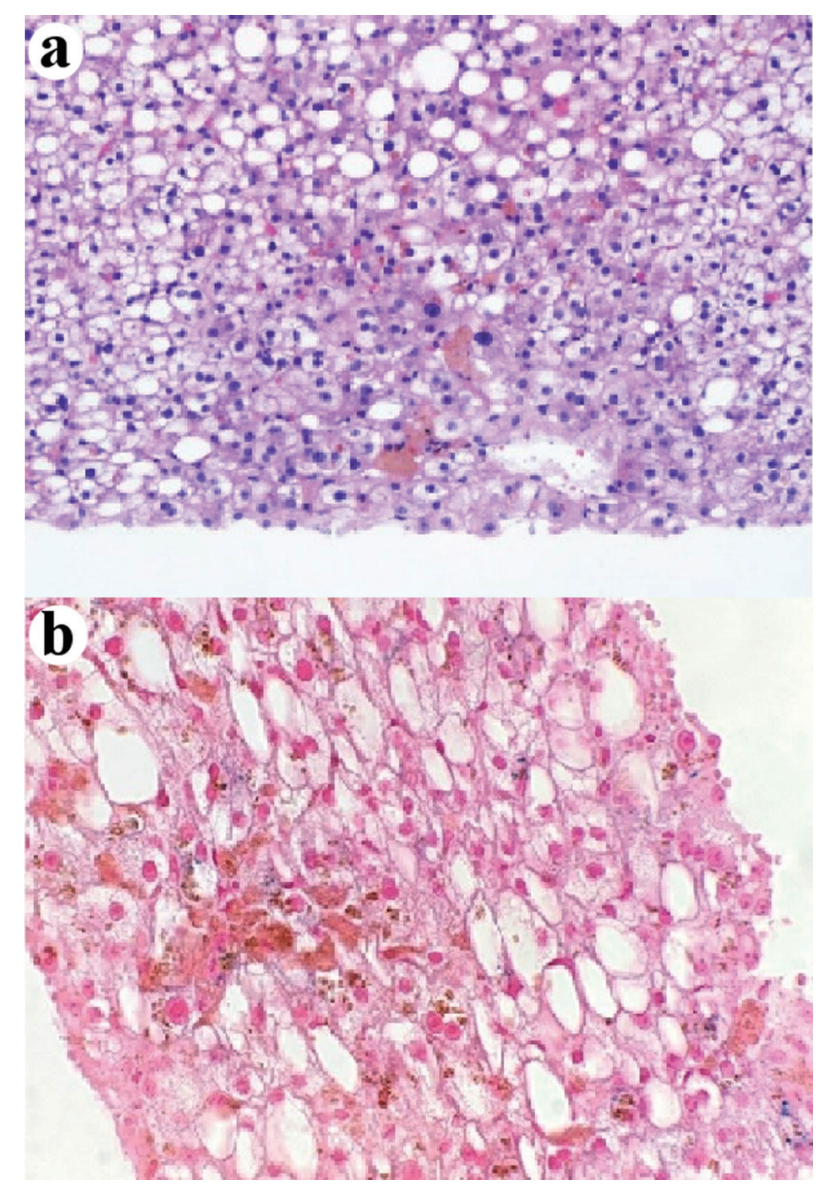

Figure 4. Centrilobular intra-hepatocellular brown pigment is negative for iron (a) H\&E stain, $\times 400$. (b) Prussian blue stain, $\times 400$. H\&E: hematoxylin and eosin.

from bariatric surgery to the current admission is much longer (13 years), and her Roux limb was standard (110 cm in length). Another possibility is that our patient's hepatic steatosis and liver failure was due to severe protein-calorie malnutrition (kwashiorkor) that was in turn caused by her bariatric surgery. One case of secondary kwashiorkor was reported to occur 10 years after Roux-en-Y gastric bypass [7]. The patient in that case report had similar clinical presentation, laboratory findings, and liver histology to our patient. Demographics, clinical presentation, and laboratory values in patients who developed hepatic complications after Roux-en-Y gastric bypass are summarized in Tables 2 and $3[4,5,7]$. All patients had significant weight loss and presented with wasting, anasarca, variable degrees of altered mental status, hypoalbuminemia, and hyperammonemia.

Wilson disease remains a major differential diagnosis because of her noted "encephalopathy/behavior" issues, her relatively young age, her low ceruloplasmin and copper levels, and the presence of moderate hepatic steatosis. Given the profound nutrition deficiency of this patient, the low ceruloplasmin finding is most likely a change secondary to her overall low protein level [7-9]. Tissue copper quantitation could help rule out Wilson disease in this case, but this test was not attempted as the patient died shortly after the liver biopsy was performed. Although there was mild siderosis in this biopsy, hereditary hemochromatosis is unlikely given the patient's low transferrin level. Mild siderosis in this case is likely due to her overall protein deficiency-associated liver pathology [10].

Another important differential diagnosis in this case is a urea cycle disorder. Urea cycle disorders include several hereditary diseases due to defects in the urea cycle which cause a Reye-like syndrome with potential fatal hyperammonemia in children, and histologically, the liver can show steatosis and focal glycogenosis [11-14]. Most patients with a urea cycle disorder were lean $[12,13]$. We think that a urea cycle disorder was unlikely in our patient. First, our patient was 46 years old, had a history of obesity, and currently had evidence of proteincalorie deficiency. She had no family history of neonatal death or liver disease or protein aversion in the past. While hyperammonemia and decreased BUN can be seen in urea cycle disorders; and protein malnutrition is known to cause both hyperammonemia $[15,16]$ and decreased $\mathrm{BUN}[8,9,17]$, these metabolic derangements may be due to overall decreased hepatic metabolic flux, including the urea cycle. Finally, the liver biopsy from our patient lacked glycogenosis, in contrast to the focal glycogenosis reported in patients with urea cycle disorders [11-13].

The liver biopsy findings in our current case are similar to those reported in the literature for kwashiorkor although liver histology in patients suffering from kwashiorkor has not been systematically documented in recent literature. It appears that most reported cases have large droplet macrovesicular steatosis that is most prominent in zone 1 , with gradual movement towards zone 3 as the disease progresses $[18,19]$. If fibrosis does occur in kwashiorkor, it appears to initially be a portal/ periportal process; however, reports of cirrhosis are rare [19, 20]. Sinusoidal dilatation has also been postulated to be related to this disorder in the past, although the mechanisms underlying this change are not clear [20,21]. One publication mentioned the presence of frequent binucleated hepatocytes in patients recovering from kwashiorkor; however, this may have been nonspecific regenerative change that can be seen after any significant liver injury [22]. Hemosiderin was reported in the livers from patients with kwashiorkor and in some of these livers, the iron may have amounted to as much as $5 \%$ of the dry weight of the liver [10]. Both lipofuscin and iron deposits (hemosiderin) in the hepatocytes are likely due to increased autophagy during protein malnutrition. However, one article mentions the absence of hemosiderin in such cases [20]. Studies showed enhanced accumulation of iron in the liver in a cyclic starvation/feeding animal model [23] and siderosis in emaciated animals [24]. The mechanism of siderosis in kwashiorkor is not clear. Siderosis in our current case may also be related to the patient's hypoceruloplasminemia and low copper levels $[25,26]$. In addition, the increased iron deposition in our case may be related to the patient's history of alcohol dependence prior to protein malnutrition. Prominent lipofuscinlike pigment is noted in hepatocytes in our current case which is consistent with the literature [10]. Lipofuscin-like pigment was reported in the livers of emaciated animals [24]. In addition, hepatic steatosis in our patient is consistent with findings for hepatic complications after bariatric surgery including 


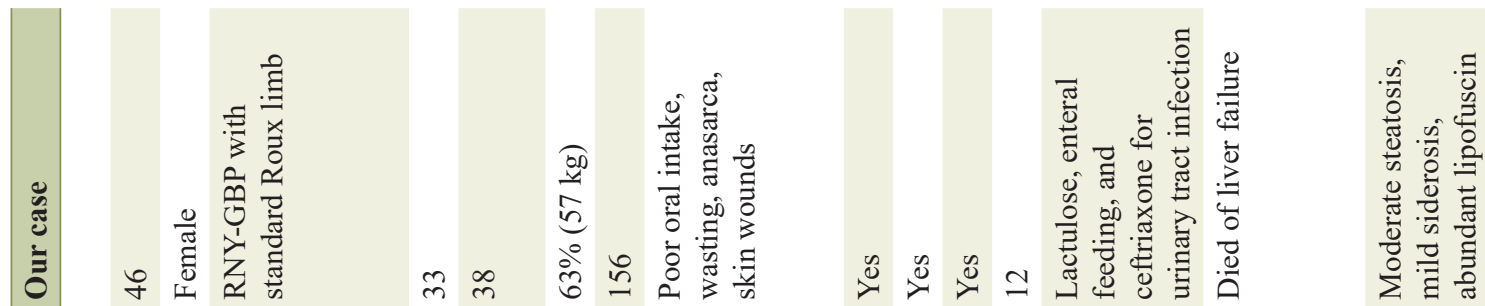

•
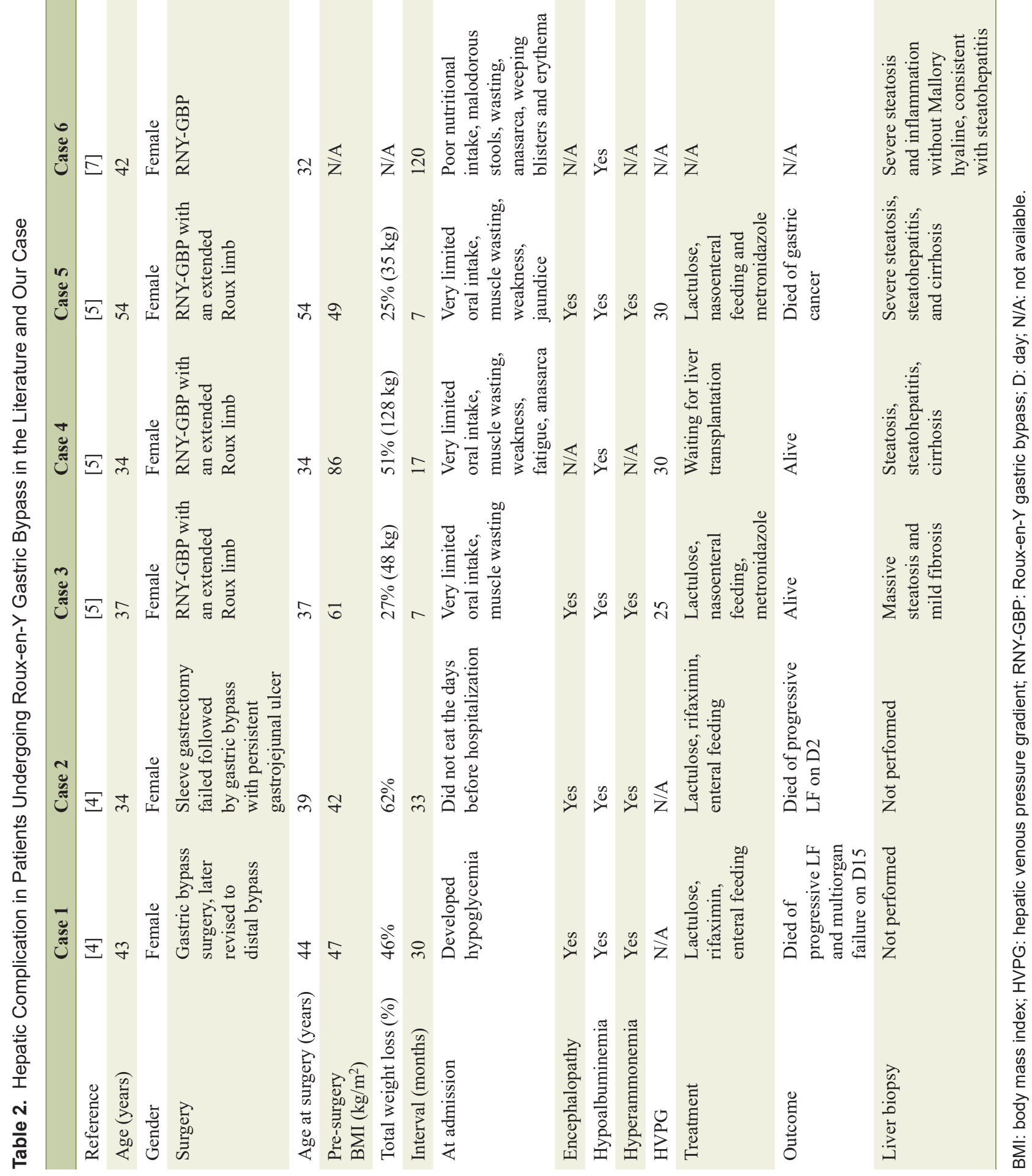
Table 3. Biochemical Abnormalities in Patients With Hepatic Complications After Roux-en-Y Gastric Bypass

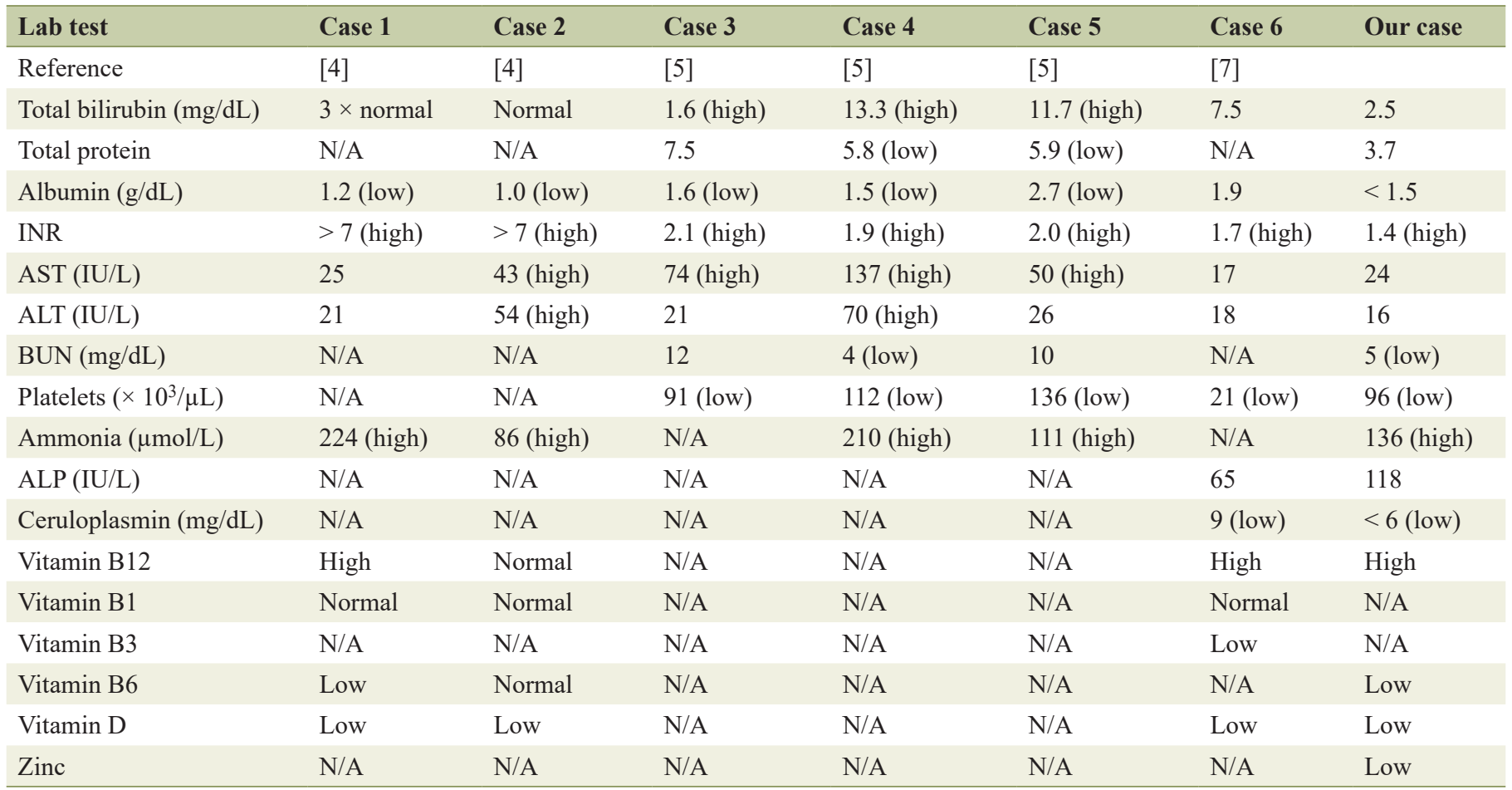

INR: international normalized ratio; AST: aspartate transaminase; ALT: alanine transaminase; BUN: blood urea nitrogen; ALP: alkaline phosphatase; N/A: not available.

Roux-en-Y gastric bypass (Table $3[4,5,7])$. In five cases with biopsies, all showed macrovesicular steatosis (at least moderate), three cases had steatohepatitis, one case had focal mild fibrosis, and two cases had cirrhosis. Although our case did not have fibrosis or cirrhosis, there was portal hypertension (HVPG of $12 \mathrm{~mm} \mathrm{Hg}$ ). An elevated HVPG of $25 \mathrm{~mm} \mathrm{Hg}$ was reported in a patient with massive steatosis and mild fibrosis [5]. The mechanism of portal hypertension in this scenario is not clear. It may be partly related to hepatic steatosis as portal hypertension has been reported in human nonalcoholic liver disease and animal models of steatosis [27-29].

Kwashiorkor is a term used for the condition characterized by a severe protein deficiency, but with an overall adequate calorie intact. Secondary kwashiorkor was reported as a late complication of gastric bypass surgery [7]. In this case, the diagnosis of kwashiorkor was made 10 years after a Roux-en-Y gastric bypass surgery. The lack of protein creates an osmotic gradient leading to edema and swelling [30]. This condition is in contrast to marasmus, which is due to overall inadequate calorie intake from all sources, not just protein. Marasmic patients will have muscular wasting and diminished subcutaneous fat. The mechanisms underlying fatty liver in patients with kwashiorkor are still not completely understood. Fatty liver is sometimes associated with marasmus as well, although typically to a lesser degree than kwashiorkor [18]. Different hypotheses have been proposed to explain steatosis in this patient population, but the true mechanism has remained elusive. Recently, an animal study demonstrated impaired mitochondrial function with steatosis in rats that were fed a low protein diet
[31]. This then led to peroxisome dysfunction, which is a possible explanation for fat accumulation in hepatocytes in patients suffering from this disease. There is suggestion that the gut microbiome may also play a role [32]. As was the case with our reported patient, many laboratory values are characteristically altered in kwashiorkor. Patients will typically have decreased total protein and albumin, abnormal electrolytes, and decreased blood sugar [33]. Cortisol and growth hormone levels are typically elevated, with decreased insulin secretion as well [33]. Iron deficiency anemia is also common in this disorder [33]. Lipid levels are also usually low, especially cholesterol [33]. However, no lipid study at this admission was performed in our patient.

Inflammatory disease, such as celiac disease, can present with protein malnutrition and hepatic steatosis, and should be included as a differential diagnosis in our case. However, the patient did not have overt diarrhea currently and EGD a few days after the admission revealed a healthy efferent limb of jejunum although jejunal biopsy was not performed.

We believe that high vigilance of protein-calorie deficiency as a potential etiology for hepatic steatosis is very important to avoid mistakenly labeling this case as alcoholic or nonalcoholic fatty liver disease. Whether kwashiorkor in this case is a late complication of a Roux-en-Y gastric bypass is not certain. Kwashiorkor is a severe disease and should be treated promptly as it carries high mortality. Even though recovery rate is expected to be greater than $75 \%$ according to the SPHERE project standards, comorbidities can affect outcomes to a significant degree [34]. However, it should also be noted 
that some other studies of treatment outcome had significantly worse outcomes than this proposed standard [34-36].

\section{Conclusions}

This case presents several relevant educational points: 1) Liver biopsy interpretation should not be attempted without the appropriate clinical context; 2) Laboratory tests should also be interpreted in the correct clinical setting; and 3) Hepatic steatosis has many etiologies including severe protein malnutrition (kwashiorkor) and bariatric surgery-associated liver failure, which each need timely and different treatment than the most common etiologies of hepatic steatosis: metabolic syndrome and alcohol use. In patients with hepatic steatosis, encephalopathy, and hyperammonemia, rare genetic diseases such as urea cycle disorders should be considered and ruled out by taking a detailed family history, asking about diet aversion, and screening with appropriate lab tests.

\section{Acknowledgments}

None to declare.

\section{Financial Declaration}

None to declare.

\section{Conflict of Interest}

None to declare.

\section{Informed Consent}

Not applicable.

\section{Author Contributions}

KNG, SD, SH drafted and critically reviewed the manuscript; $\mathrm{XL}$ originated the idea and supervised the project.

\section{Data Availability}

The authors declare that data supporting the findings of this study are available within the article.

\section{References}

1. Lagana SM, Kudose S, Iuga AC, Lee MJ, Fazlollahi L, Remotti HE, Del Portillo A, et al. Hepatic pathology in patients dying of COVID-19: a series of 40 cases includ- ing clinical, histologic, and virologic data. Mod Pathol. 2020;33(11):2147-2155.

2. Anstee QM, Day CP. Epidemiology, natural history, and evaluation of nonalcoholic fatty liver disease. In: Sanyal AJ, Boyer TD, Lindor KD, Terrault NA (Eds). Zakim and Boyer's Hepatology. 7th edition. Elsevier, Philadelphia, 2018. p. 391-405.

3. Maxwell JD, Sanderson I, Butler WH, Gazet JC, Pilkington TR. Hepatic structure and function after modified jejunoileal bypass surgery for obesity. Br Med J. 1977;2(6089):726-729.

4. Lammers WJ, van Tilburg AJ, Apers JA, Wiebolt J. Liver failure caused by prolonged state of malnutrition following bariatric surgery. World J Hepatol. 2018;10(3):396399.

5. Cotler SJ, Vitello JM, Guzman G, Testa G, Benedetti E, Layden TJ. Hepatic decompensation after gastric bypass surgery for severe obesity. Dig Dis Sci. 2004;49(10):15631568.

6. Schauer PR, Bhatt DL, Kirwan JP, Wolski K, Aminian A, Brethauer SA, Navaneethan SD, et al. Bariatric Surgery versus Intensive Medical Therapy for Diabetes - 5-Year Outcomes. N Engl J Med. 2017;376(7):641-651.

7. William JH, Tapper EB, Yee EU, Robson SC. Secondary kwashiorkor: a rare complication of gastric bypass surgery. Am J Med. 2015;128(5):e1-2.

8. Verjee ZH, Behal R. Protein-calorie malnutrition: a study of red blood cell and serum enzymes during and after crisis. Clin Chim Acta. 1976;70(1):139-147.

9. Mauron J, Antener I. Is the adult protein-energy malnutrition syndrome the same as that described in the infant? Experientia Suppl. 1983;44:298-338.

10. Gillman J, Gillman T, Mandelstam J, Gilbert C. The production of severe hepatic injury in rats by the prolonged feeding of maize-meal porridge (mealie-pap) and sour milk. Br J Exp Pathol. 1945;26:67-81.

11. Badizadegan K, Perez-Atayde AR. Focal glycogenosis of the liver in disorders of ureagenesis: its occurrence and diagnostic significance. Hepatology. 1997;26(2):365-373.

12. Ranucci G, Rigoldi M, Cotugno G, Bernabei SM, Liguori A, Gasperini S, Goffredo BM, et al. Chronic liver involvement in urea cycle disorders. J Inherit Metab Dis. 2019;42(6):1118-1127.

13. Bigot A, Tchan MC, Thoreau B, Blasco H, Maillot F. Liver involvement in urea cycle disorders: a review of the literature. J Inherit Metab Dis. 2017;40(6):757-769.

14. Shenoy A, Wilkins BJ, Russo P. Childhood liver diseases and metabolic disorders. In: Liu, Lai, Jhala (Eds). Practical Interpretation of Liver Biopsy. Volume 1. Cambridge Scholars, 2020.

15. Kinnear AA, Pretorius PJ. Liver function in kwashiorkor. Br Med J. 1956;1(4982):1528-1530.

16. Abo-Hussein SA, Hussein ZM, Farag SI, Shebl SS, elMelegy S, Akhnoukh S. A profile of ammonia-urea values in blood and cerebrospinal fluid in children with protein energy malnutrition. J Trop Med Hyg. 1984;87(6):237240.

17. Anthony LE, Edozien JC. Experimental protein and energy in the rat. J Nutr. 1975;105(6):631-648. 
18. Bailey KV. Liver pathology in malnourished New Guineans. Med J Aust. 1966;1(14):572-575.

19. Davies JN. The pathology of dietary liver disease in tropical Africa. Ann N Y Acad Sci. 1954;57(6):714-721.

20. Chanda NK. Pathological study of the liver in kwashiorkor. Br Med J. 1958;1(5082):1263-1266.

21. Doherty JF, Adam EJ, Griffin GE, Golden MH. Ultrasonographic assessment of the extent of hepatic steatosis in severe malnutrition. Arch Dis Child. 1992;67(11):13481352.

22. Cook GC, Hutt MS. The liver after kwashiorkor. Br Med J. 1967;3(5563):454-457.

23. Richter GW. Effects of cyclic starvation-feeding and of splenectomy on the development of hemosiderosis in rat livers. Am J Pathol. 1974;74(3):481-506.

24. Josefsen TD, Sorensen KK, Mork T, Mathiesen SD, Ryeng KA. Fatal inanition in reindeer (Rangifer tarandus tarandus): pathological findings in completely emaciated carcasses. Acta Vet Scand. 2007;49:27.

25. Matsushima A, Yoshida T, Yoshida K, Ohara S, Toyoshima Y, Kakita A, Ikeda S. Superficial siderosis associated with aceruloplasminemia. Case report. J Neurol Sci. 2014;339(1-2):231-234.

26. Doyle A, Rusli F, Bhathal P. Aceruloplasminaemia: a rare but important cause of iron overload. BMJ Case Rep. 2015;2015:bcr2014207541.

27. Francque S, Verrijken A, Mertens I, Hubens G, Van Marck E, Pelckmans P, Van Gaal L, et al. Noncirrhotic human nonalcoholic fatty liver disease induces portal hypertension in relation to the histological degree of steatosis. Eur J Gastroenterol Hepatol. 2010;22(12):1449-1457.

28. Mendes FD, Suzuki A, Sanderson SO, Lindor KD, Angulo P. Prevalence and indicators of portal hypertension in patients with nonalcoholic fatty liver disease. Clin Gastroenterol Hepatol. 2012;10(9):1028-1033 e1022.

29. Baffy G. Origins of Portal Hypertension in Nonalcoholic Fatty Liver Disease. Dig Dis Sci. 2018;63(3):563-576.

30. Coulthard MG. Oedema in kwashiorkor is caused by hypoalbuminaemia. Paediatr Int Child Health. 2015;35(2): 83-89.

31. van Zutphen T, Ciapaite J, Bloks VW, Ackereley C, Gerding A, Jurdzinski A, de Moraes RA, et al. Malnutritionassociated liver steatosis and ATP depletion is caused by peroxisomal and mitochondrial dysfunction. J Hepatol. 2016;65(6):1198-1208.

32. Smith MI, Yatsunenko T, Manary MJ, Trehan I, Mkakosya R, Cheng J, Kau AL, et al. Gut microbiomes of Malawian twin pairs discordant for kwashiorkor. Science. 2013;339(6119):548-554.

33. Benjamin DR. Laboratory tests and nutritional assessment. Protein-energy status. Pediatr Clin North Am. 1989;36(1):139-161.

34. Derseh B, Mruts K, Demie T, Gebremariam T. Comorbidity, treatment outcomes and factors affecting the recovery rate of under -five children with severe acute malnutrition admitted in selected hospitals from Ethiopia: retrospective follow up study. Nutr J. 2018;17(1):116.

35. Saaka M, Osman SM, Amponsem A, Ziem JB, AbdulMumin A, Akanbong P, et al. Treatment outcome of severe acute malnutrition cases at the Tamale Teaching Hospital. J Nutri Metab. 2015;2015:1-8.

36. Kabalo MY, Seifu CN. Treatment outcomes of severe acute malnutrition in children treated within Outpatient Therapeutic Program (OTP) at Wolaita Zone, Southern Ethiopia: retrospective cross-sectional study. J Health Popul Nutr. 2017;36(1):7. 